other than those desired in the mechanism are prevented." When one of the mechanical forces of nature, such as that of falling water, moving air, or expanding steam, is applied to one of the movable links in such a manner as to cause it to change its position, mechanical work is performed accompanied by certain determinate motions, and the whole is called a machine. The relative motion of two bodies in a plane is next considered, and the conceptions of the instantaneous centre and of centroids

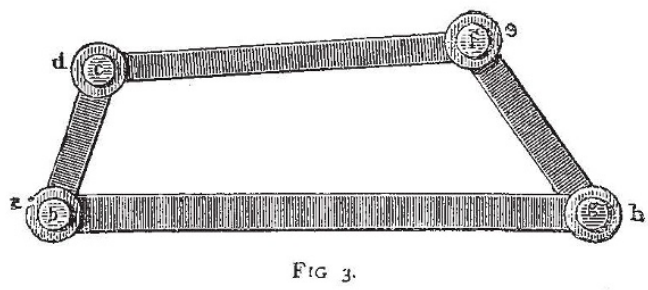

introduced. At each instant of the motion in a plane of one body witk respect to another considered as fixed, the motion can be accurately represented by a rotation in the plane about a fixed point, which, however, in each succeedin $\sigma$ instant may occupy a different position; this point is called the instantaneous centre, and the positions it occupies in successive instants trace the centroid. Space will not permit us to show the formation of the reciprocal centroid, or how the motion of the moving body can be represented at each instant by the rolling on one another of the centroids, and the motion of any points connected rigidly with the moving body determined when the centroids are known, but the example given as illustrating the determination of relative motion from the known centroids will speak for itself. The centroids given are a circle and straight line which roll on each other. All points rigidly connected with the circle

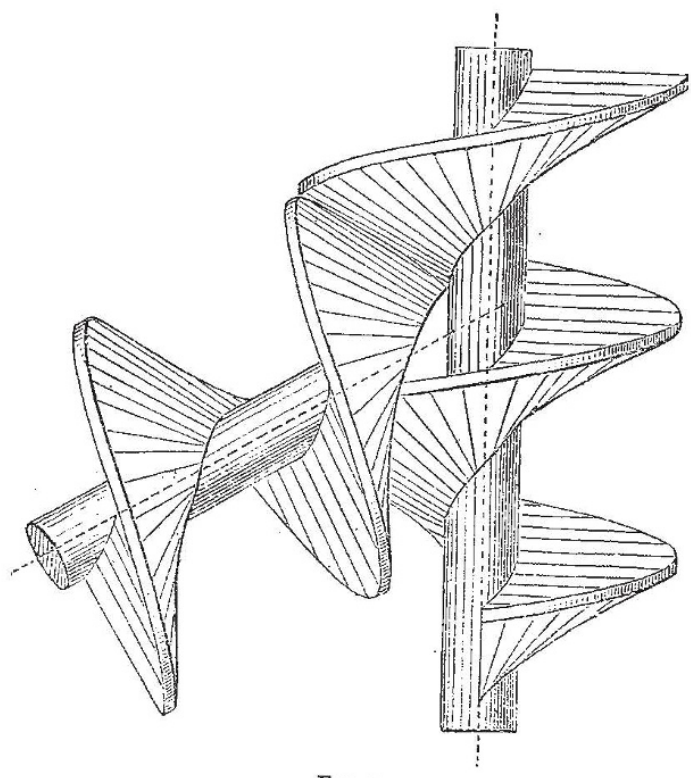

FIG. 4.

describe trochoids, the line being regarded as fixed; all points rigidly connected with the straight line describe involutes, the circle being considered fixed, and all these paths are determinate, and can be constructed if the position of the moving point with respect to the moving element, circle or line, and the centroids, the line and circle are given.

From motion in a plane and the determination of centroids. we pass to motion in space. If the position of three points in a rigid body not in the same straight line are known, the position of any other point in it may be determined from them, and if the three points are fixed in space the body is also fixed. Thus, to determine the relative motion of two rigid bodies in space, we have only to consider the motion of two triangles fixed one in each of them ; or the motion of one triangle fixed in the moving body with respect to the other reduced to rest. The change of position of the moving triangle may take place in many ways, but it may in every case be effected by its translation parallel to itself in a line joining the old and new positions of one of its angular points, and then by a rotation about an axis through the new position of that angular point. Thus any change of position of a rigid body may be effected by a simple translation and a simple translation about an axis. The simplest case is when the transiation takes place along a line parallel to the axis of rotation, when, if the change of position of the moving body be taken indefinitely small, the instantaneous axes of rotation along which sliding simultaneously takes place become indefinitely near each other; the motion is then a simple twist.

"Consider a pair of bodies having conical rolling, in which both cones have a motion of translation in space. The rotation then takes place through the conical rolling, and the sliding through the translation of the pair of bodies." Next "consider the consecutive positions of the axes as forming a pair of ruled surfaces, one for each body, so that the motion is reduced to the rolling of the two ruled surfaces upon each other with a simultaneous endlong sliding upon each other of the generators which are in contact. The surfaces of these solids being the loci of the axes, are called axoids. Thus all relative motions of two bodies may be considered as the twisting or rolling of ruled surfaces or axoids." The ruled surfaces roll on each other without sliding, when all the axes meet in a point as in a pair of cones or a cone and cylinder; also when the point of intersection is at any infinite distance, as in the case of two cylinders with parallel axes. These are, however, only particular and more obvious cases of the general condition of rolling without sliding, viz., that the two ruled surfaces are developable on each other.

\section{APPARATUS FOR REGISTERING ANIMAL MOVEMENTS 1}

THE registering apparatus which have enabled us to carzy so far the investigation of the functions in living animals are applicable to the analysis of movements of every kind in health and in disease. It is to this important application that I desire to draw your attention at the present time.

Most of the movements whose various phases we have to estimate must be transmitted to a distance, preserving at the same time all their characteristics. It is by the medium of the air that this transmission is effected, and its principle is as follows :-

Upon the organ (muscle, artery, heart) whose movements are to be investigated an apparatus called the exploring drum is applied. It is a small metal basin closed by a caoutchouc membrane, and communicating by a longer or shorter tube with a similar drum, upon the membrane of which is supported a recording lever. The pen with which the extremity of this lever is provided inscribes the curve of the movement impressed on the membrane of the first drum on a cylinder covered with smoked paper and turning on a horizontal axis.

I. Let us at once apply the process of analysis to the muscular movements of man. For this purpose we may either grasp the muscles of the ball of the thumb between the flattened jaws of the pincers which I show you, or apply to the fleshy substance of any muscle an exploring drum, the knob of which rests upon the muscle. When by means of electricity we cause a contraction or tetanus of the muscle to be studied, the curve of the contraction or that of the tetanus is recorded at a distance upon the revolving cylinder.

This apparatus shows the thickening which a muscle undergoes I Paper read in the Biological Section at the Loan Collection Conferences, by Prof. Marey. 
during contraction, and it furnishes results identical to those obtained by investigating the shortening of the muscle during contraction in living animals. We are then quite authorised to interpret in the same way the curves obtained in both cases.

It is needless to insist on the numerous services which the myography of man may render to physiology and medicine. The study of the forms of movement, of the latent period, of muscie, and perhaps even tive rate of transmission of impulses along motor nerves, by means of this apparatus may be as easily pursued in healthy or unhealthy men as in animals.

2. Without quitting the investigation of muscular movements, let us examine that of the respiratory movements, and we shall obtain valuable information as to the means by which the important function of respiration is effected. We apply to the chest this apparatus formed of an elastic plate and furnished with two lever arms, to the extremity of which is attached a band which surrounds the thorax. Each dilatation of the chest causes the spring to bend, and it resumes its position during respiration. This double movement is accompanied by a rising and falling of the membrane of the drum which forms part of the apparatus, and which therefore becomes a regular bellows, causing the elevation and depression of the inscribing lever placed beside the cylinder.

The respiratory curves thus obtained present certain normal characteristics susceptible of being greatly modified when any obstruction interferes with the respiratory functions either by impeding the entrance or the exit of the air, or even by opposing its passage in both directions. In all these cases the curves have a special physiognomy, and their simple inspection enables us to recognise the seat of the obstacle to respiration. Clinical research will yet discover here many points for investigation.

3. But above all there are the phenomena of circulation, which have been minutely investigated both in man and animals. The apparatus by means of which we can completely analyse the movement of the heart, the arterial pulse, \&c., have aiready rendered great service; we are, however, right in expecting yet more from it, by making use of it in clinical investigations.

Of various cardiographs, that on which I wish to dwell differs litule from the explorer of which I have already spoken. The knob with which it is provided is applied to the region of the apex of the heart, and each beat of the organ is transmitted to the recording lever. There is seen in this pulsation of the heart the same elements which the physiological cardiograph has revealed in the higher animals. This beating of the heart is then a complex act, and the numerous details which have been discovered by graphic analysis have each a considerable importance from the point of view of functional investigation. One part of the tracing shows us how the ventricle is emptied into the artery; another enables us to appreciate the play of the auricle, the beating of the sigmoid valves, \&c. You will easily see that the precise diagnosis of affections of the heart, already carried so far, thanks to auscultation, will be greatly improved by the application to man of the cardiograph applied to the study of the pulsation of the heart.

The arterial pulse cannot be separated from the pulsation of the heart in the study of the pheromena of circulation in man. Already numerous researches have been undertaken by means of the direct sphygmograph; but much more may be expected from the use of the air sphygmograph (sphygmographe a transmission).

I place this apparatus upon my wrist, and the artery raising a spring connected with the membrane of the exploring drum, transmits its mavement to a distance by means of the tube filled with air, which enables this sphygmograph to communicaie with the drum to which the recording lever is attached. By recording simultaneously the traces of the pulse and those of the heart much information may be obtained and many errors avoided.

4. I shall present to you, in conclusion, a new method of investigating the peripheral circulation. This method is based on the principle that the variation of the calibre of the bloodvessels in any part of the body is faithfully indicated by the variations of the volume of that part. Without dwelling on the history of these investigations, I may tell you that they originated many years ago, Dr. Piégu, of Paris, having pointed out in 1846 the alternate expansion and contraction of the tissues in connection with the dilatation and contraction of the blood-vessels. Since that time Chelius and Fick in Germany, Mosso in Italy, Franck at Paris, have carried on and extended these researches.

The recording of the movements of a column of water inclosed in a tube communicating with a receiver filled with water and into which the hand and forearm is plunged, was first effected. by Fick by means of a float armed with a pen. Ch. Buisson hit on the happy idea of transmitting to a distance, by means of tubes filled with air, the oscillation of the column of water, and it is with his apparatus that M. Franck, in my laboratory, has execuled a series of researches. You see the apparatus in action. The hand is plunged into this jar filled with water ancl hermetically closed. A vertical tube, furnished with a bulb to avoid the effects of the speed acquired by the liquid, serves tis transmit to a recording lever the oscillation of the column of water. You will remark that these oscillations are rhythmica with the heart, and if we record them by the side of the cardias pulse registered by the transmitting sphygmograph, we can establish the identity of the variations in size or, as we may term them, the pulsations of the hand and of the pulsations of a single artery. With this apparatus we may perform numerous experi. ments on the mechanical effects of compression of the arteries or veins, the action of the vaso-motor system of nerves, direct ot reflex, \&c.

I shall not explain to you by the side of this method of investigation, that which we owe to Mosso, of Turin. His plethysmograph, which ought soon to be presented to you, permits the estimation of changes of volume of the hand, and, assuredly, the combination of these two processes ought to lead to importan results in the investigation of the phenomena of peripheral circulation.

I have sought to submit to you some of the points more immediately applicable to man, without dwelling on the investigation of the movements among animals. But these two orders of researches complement each other. We may say that most of the data furnished by experimentation on animals are now susceptible of rigorous verification on man, healthy or unhealthy. This verification we owe to investigation by means of precise apparatus and to the recording of the smallest movements, thanks to the registering instruments, the principal specimens of which are shown in this Collection.

\section{DREDGINGS OF THE "CHALLENGER"}

PROF. WYVILLE THOMSON had not set foot long in Old England before presenting in person a preliminary quota of his results to the learned bodies. Two papers read by him at the Linnean Society on June I, embodied observations on Echinodermata, a group to which, as is well known, he previously had paid much attention. One of the communications described some new living Crinoids belonging to the Apiocrinidx. Of deep-sea forms the stalked crinoids are extremely rare, and have a special interest on account of their palæontological relations; it was therefore with satisfaction that near St. Paul's rocks at 1,850 fathoms, the trawl brought up, among other things, an entire specimen of a new crinoid, Bathycriniss Aldrichianus, and fragments of another, Hyocrinus bethellianus. At other stations and on different occasions, were obtained another species of Bathycrinus (B. gracilis) and an undetermined beautiful little species of Hyocrinus, besides examples of the Rhizocrinus lofotensis of Sars; all of these being referable to the Apiocrinidæ. In pointing out their structural peculiarities and alluding to Bathycrinus, he mentioned that the stem barely enlarges at its junction with the cup, the ring formed by the basals is very small, and the first radiais are free from the basals and often free from one another, while the oral plaies are absent. This gents appears to possess an assemblage of characters in some respects intermediate between Rhizocrinus and the pentacrinoid stage of Antedon. Hyocrinus bethellinnus has much the appearance, and in some prominent particulars it seems to have very much the structure of the palæozoic genus Platycrinus or its sub-genus Dichocrinus. The stem is much more rigid than that of Bathycrinus; the cup consists of two tiers of plates only, the lower is to be regarded as a ring of basals, and the upper consists of fine spade-shaped radials. There are five arms which are pinnulated. The proximal pinnules are very long, running on nearly to the end of the arm, and the succeeding pinnules are gradually shorter, all of them, however, running out to the end of the arm. I istaily the ends of the five arms, and the ends of all the pinnules meet nearly on a level. Thi; arrangement is unknown in recent crinoids, although we have something close to it in species of the fossil genera Poteriocrinus and Cyathocrinus; with this, however, their resemblances end. Rhizocronus finds its ally in the cretaceous genus Bourgueticrinus; Bathycrinus and Hyocrinus are evidently related to the former, but the characters of the Apiocrinidæ are nevertheless obscure in 